\title{
Ermine phenotype
}

INSERM

\section{Source}

INSERM. (1999). Orphanet: an online rare disease and orphan drug data base. Ermine phenotype. ORPHA:999

Cutaneous albinism-ermine phenotype is characterised by the association of white hair with black tufts, depigmented skin and sensorineural deafness. It has been described in two pairs of siblings and one individual case. The depigmentation may present as vitiligo, or be spotted with brown patches. Nystagmus, photophobia, retinal depigmentation and intellectual deficit were also reported in one pair of siblings. An autoimmune mechanism or failure of melanocyte migration may be responsible for the disease. 